\title{
MicroRNA-661 promotes non-small cell lung cancer progression by directly targeting RUNX3
}

\author{
YU WANG*, YUQIANG LI*, BIN WU*, CE SHI and CHEN LI \\ Department of Molecular Detection, Center for Clinical Biological Samples, The First Affiliated \\ Hospital of Jinzhou Medical University, Jinzhou, Liaoning 121001, P.R. China
}

Received February 12, 2017; Accepted June 8, 2017

DOI: $10.3892 / \mathrm{mmr} .2017 .6827$

\begin{abstract}
Lung cancer is the primary cause of cancer-associated mortality in men and women worldwide. Increasing evidence indicates that abnormal microRNA (miRNA) expression contributes to the carcinogenesis and progression of multiple human cancers, including non-small cell lung cancer (NSCLC). Therefore, miRNAs exhibit the potential to act as biomarkers for the diagnosis, treatment and prognosis of human malignancies. miRNA-661 (miR-661) has previously been demonstrated to be important in the development of various human cancer types. However, the expression levels, functions and underlying mechanisms of miR-661 in NSCLC remain to be elucidated. The present study demonstrated that miR-661 was upregulated in NSCLC tissues and cell lines. In addition, miR-661 expression levels were significantly correlated with differentiation and tumor stage lymph node metastasis of NSCLC patients. Functional experiments demonstrated that miR-661 downregulation inhibited NSCLC cell proliferation and invasion in vitro. Furthermore, runt-related transcription factor 3 (RUNX3) was identified as a direct target of miR-661 in NSCLC. RUNX3 was expressed at a low level in NSCLC tissues and was negatively correlated with the miR-661 expression level. Further experiments revealed that RUNX3 knockdown significantly rescued the effects of miR-661 underexpression on NSCLC cell proliferation and invasion. In conclusion, the present findings indicated a role for miR-661 as an oncogene in NSCLC via direct targeting of RUNX3, thus suggesting that miR-661 may be used to develop novel therapies for NSCLC patients.
\end{abstract}

Correspondence to: Professor Chen Li, Department of Molecular Detection, Center for Clinical Biological Samples, The First Affiliated Hospital of Jinzhou Medical University, 2 Renmin Road, Jinzhou, Liaoning 121001, P.R. China

E-mail: lichen_jinzhou@126.com

*Contributed equally

Key words: non-small cell lung cancer, microRNA-661, runt-related transcription factor 3, progression, proliferation, invasion

\section{Introduction}

Lung cancer is the most common malignancy in humans and is the primary cause of cancer-related mortality in both men and women globally $(1,2)$. Previous studies demonstrated that accumulation of permanent genetic alterations in combination with dynamic epigenetic alterations results in lung cancer formation and progression $(3,4)$. Lung cancer can be divided into two major groups based on pathological features: Small cell lung cancer (SCLC) and non-small cell lung cancer (NSCLC) (5). NSCLC is clinically characterised by rapid progression, strong invasiveness and high mortality rate (6). NSCLC accounts for $\sim 85 \%$ of all lung cancer cases and includes squamous cell carcinoma, adenocarcinoma, and large cell carcinoma (7). Although considerable progress in surgery, diagnostic method, radiotherapy and new chemotherapy regimens has been made, the prognosis of NSCLC patients remains poor (8). Recurrence and metastasis, even after radiation therapy and/or chemotherapy, are the major causes of death among NSCLC patients (5). Therefore, further understanding of the underlying molecular mechanisms during NSCLC initiation and progression is necessary. Moreover, the development of novel efficient therapeutic strategies for patients with this malignancy is urgently needed.

MicroRNAs (miRNAs) are a large group of endogenous, single-strand, non-coding and short RNAs that are between approximately 19 and 25 nucleotides in length (9). MiRNAs serve as key regulators of gene expression through direct binding to the 3'-untranslated region (UTR) of their target genes in a sequence-specific manner, leading to either translation inhibition or messenger RNA (mRNA) degradation (10). In recent years, miRNAs have emerged as powerful regulators of various physiological and pathological processes, such as growth, apoptosis, differentiation, angiogenesis, inflammation and tumourigenesis (11). Increasing evidence has shown that more than half of miRNA genes are located at fragile sites (12-14). Several studies reported that numerous miRNAs are aberrantly expressed in various human cancers and play significant roles in tumourigenesis and tumour development, including NSCLC (15-17). Obviously, miRNAs can function as either tumour suppressors or oncogenes in different human cancers depending on the characteristics of their target genes (18). Therefore, miRNAs are potential biomarkers for diagnosis, treatment and prognosis of human malignancies 
owing to their tissue- and disease-specific expression and regulatory functions (19).

MicroRNA-661 (miR-661) has been reported to be abnormally expressed in several tumours (20-22). However, the miR-661 expression and functions in NSCLC are yet to be investigated. The present study aims to elucidate the miR-661 expression, clinical significance and biological roles in NSCLC and to investigate its underlying molecular mechanisms.

\section{Materials and methods}

Tissue samples. Forty-seven pairs of NSCLC tissues and adjacent non-cancer tissues were obtained from patients who had undergone surgical resection at The First Affiliated Hospital of Jinzhou Medical University (Liaoning, China) between February 2013 and June 2015. All of these patients had not been treated with any pre-operative cancer treatment, such as radiotherapy or chemotherapy. All tissues were rapidly snap-frozen in liquid nitrogen and stored at $-80^{\circ} \mathrm{C}$ for subsequent experiments. The protocol of the present study was reviewed and approved by the Ethics Committee of The First Affiliated Hospital of Jinzhou Medical University. Written informed consent was also provided by all patients who enrolled in the present study.

Cell lines, culture and transfection. Non-tumorigenic bronchial epithelium BEAS-2B cells and four NSCLC cell lines (A549, H460, SK-MES-1 and SPC-A1) were purchased from Shanghai Institute of Biochemistry and Cell Biology (Shanghai, China). BEAS-2B cells were cultured in LHC-9 medium (Gibco; Thermo Fisher Scientific, Inc., Waltham, MA, USA) supplemented with $10 \%$ fetal bovine serum (FBS; Gibco; Thermo Fisher Scientific, Inc.). NSCLC cell lines were maintained in Dulbecco's Modified Eagle's Medium (DMEM; Gibco; Thermo Fisher Scientific, Inc.) containing 10\% FBS and $1 \%$ penicillin-streptomycin (Sigma Aldrich, Saint Louis, $\mathrm{MO}, \mathrm{USA})$. All cells were grown at $37^{\circ} \mathrm{C}$ in a humidified atmosphere with $5 \% \mathrm{CO}_{2}$.

MiR-661 inhibitor and corresponding scramble miRNA inhibitor negative control (NC inhibitor) were synthesized by GenePharma (Shanghai, China). Small interfering RNA (siRNA) targeting RUNX3 (si-RUNX3) and an unrelated sequence used as a negative control of siRNA (si-NC) were obtained from Ribobio Technology Co., Ltd. (Guangzhou, China). Cells were collected, counted and seeded into 6-well plates the day before transfection. Cell transfection was performed using lipofectamine 2000 (Invitrogen, Carlsbad, CA, USA), in accordance with the manufacturer's protocol. DMEM with $10 \%$ FBS was added to each well at $6 \mathrm{~h}$ following transfection.

RNA isolation and reverse transcription-quantitative polymerase chain reaction $(R T-q P C R)$. Total RNA was isolated from tissues or cells with TRIzol reagent (Invitrogen; Thermo Fisher Scientific, Inc., Waltham, MA, USA) following to the manufacturer's protocols. The concentration of the total RNA was examined using the ND-2000 spectrophotometer (NanoDrop Technologies; Thermo Fisher Scientific, Inc., Pittsburgh, PA, USA). For the analysis of miR-661 expression, RNA was reverse-transcribed to cDNA using a TaqMan ${ }^{\circledR}$ MicroRNA Reverse Transcription kit (Applied Biosystems; Thermo Fisher Scientific, Inc., Waltham, MA, USA) and
qPCR was carried out using the TaqMan MicroRNA Assay kit (Applied Biosystems; Thermo Fisher Scientific, Inc.) on an Applied Biosystems 7500 Real-time PCR System (Thermo Fisher Scientific, Inc.). For quantification of RUNX3 mRNA, cDNA was synthesized using PrimeScript ${ }^{\mathrm{TM}}$ RT Reagent kit (Takara Biotechnology Co., Ltd., Dalian, China), followed by qPCR with SYBR Premix Ex Taq mastermix (Takara Biotechnology Co, Ltd.). RUN6B and GAPDH were used as internal control for miR-661 and RUNX3 mRNA, respectively. The primers used in the present study were as follows: miR-661 forward, 5'-GTGCCTGGGTCTCTGGCCT-3', and reverse, 5'-CGTCATGATGTTGCGTCACC-3'; RUN6B forward, 5'-CTCGCTTCGGCAGCACATATACT-3', and reverse, 5'-ACGCTTCACGAATTTGCGTGTC-3'. RUNX3 forward, 5'-GACAGCCCCAACTTCCTCT-3', and reverse, 5'-CACAGTCACCACCGTACCAT-3'; GAPDH forward, 5'-GGGTGTGAACCATGAGAAGT-3', and reverse, 5'-GAC TGTGGTCATGAGTCCT-3'. The $2^{-\Delta \Delta C t}$ method was used to calculate the relative expression of miRNA and mRNA (23).

Cell Counting Kit-8 (CCK-8) assay. Cell proliferation was measured with CCK8 assay (Dojindo Laboratories, Kumamoto, Japan). Cells were collected and seeded into 96-well plates at a density of 3000 cells per well the day before transfection. Cells were then transfected with miR-661 inhibitor, NC inhibitor, si-RUNX3 or si-NC. Subsequent to being incubated at $37^{\circ} \mathrm{C}$ in a $5 \% \mathrm{CO}_{2}$ incubator for $0-72 \mathrm{~h}, 10 \mu \mathrm{l}$ CCK-8 reagent was added into each well and incubated at $37^{\circ} \mathrm{C}$ for additional $4 \mathrm{~h}$. The optical density (OD) at $450 \mathrm{~nm}$ for each well was determined using an ELISA reader (Bio-Rad Laboratories, Inc., Hercules, CA, USA). All experiments were repeated in triplicate.

Cell invasion assay. Cell invasion assays were performed using Transwell chambers (pore size, $8 \mathrm{~mm}$; Millipore Corp., Billerica, MA, USA) pre-coated with Matrigel (BD Biosciences, Bedford, MA, USA). Transfected cells were harvested at $48 \mathrm{~h}$ post-transfection, re-suspended in FBS-fee DMEM and then seeded into the upper Transwell chambers. In the lower chamber, DMEM containing 10\% FBS was added. After $48 \mathrm{~h}$ of incubation at $37^{\circ} \mathrm{C}$ with $5 \% \mathrm{CO}_{2}$, the non-invasive cells were removed carefully with cotton swabs. The cells that had invaded through the chamber membrane were fixed with $4 \%$ paraformaldehyde and stained with $0.1 \%$ crystal violet. Subsequent to washing with PBS, cells in five randomly selected visual fields were photographed and counted under an inverted microscope (Olympus Corporation, Tokyo, Japan).

miRNA target prediction. Human miRNA target prediction algorithms: PicTar (http://pictar.mdc-berlin.de/) and TargetScan (http://www.targetscan.org/) were used to predicate the potential targets of miR-661.

Luciferase reporter assays. For reporter assays, the human RUNX3 wild type or mutated 3'-UTR sequence containing the miR-661 binding site was inserted into psiCHECK-2 vector to develop psiCHECK2-RUNX3-3'UTR-Wt and psiCHECK2-RUNX3-3'UTR-Mut. Cells were seeded into 24 -well plates at $5 \times 10^{4}$ cells per well and transfected with miR-661 inhibitor or NC inhibitor, together with psiCHECK2-RUNX3-3'UTR-Wt or 
Table I. Association between microRNA-661 expression and clinicopathologic factors of non-small cell lung cancer.

\begin{tabular}{|c|c|c|c|c|}
\hline \multirow{2}{*}{$\begin{array}{l}\text { Clinicopathological } \\
\text { factors }\end{array}$} & \multirow[b]{2}{*}{ Cases } & \multicolumn{2}{|c|}{$\begin{array}{c}\text { microRNA- } \\
661 \\
\text { expression }\end{array}$} & \multirow[b]{2}{*}{ P-value } \\
\hline & & High & Low & \\
\hline Gender & & & & 0.642 \\
\hline Male & 20 & 11 & 9 & \\
\hline Female & 27 & 13 & 14 & \\
\hline Age (years) & & & & 0.676 \\
\hline$<60$ & 19 & 9 & 10 & \\
\hline$\geq 60$ & 28 & 15 & 13 & \\
\hline Smoker & & & & 0.440 \\
\hline Yes & 28 & 13 & 15 & \\
\hline No & 19 & 11 & 8 & \\
\hline Tumor size $(\mathrm{cm})$ & & & & 0.423 \\
\hline$<3$ & 25 & 12 & 13 & \\
\hline$\geq 3$ & 22 & 12 & 10 & \\
\hline Differentiation & & & & $0.011^{\mathrm{a}}$ \\
\hline Moderate-Well & 28 & 10 & 18 & \\
\hline Poorly & 19 & 14 & 5 & \\
\hline Tumor stage & & & & $0.013^{\mathrm{a}}$ \\
\hline I-II & 24 & 8 & 16 & \\
\hline III-IV & 23 & 16 & 7 & \\
\hline Lymph node metastasis & & & & $0.029^{\mathrm{a}}$ \\
\hline Negative & 21 & 7 & 14 & \\
\hline Positive & 26 & 17 & 9 & \\
\hline
\end{tabular}

${ }^{\mathrm{a}} \mathrm{P}<0.05$.

psiCHECK2-RUNX3-3'UTR-Mut. Following incubation at $37^{\circ} \mathrm{C}$ for $48 \mathrm{~h}$, the Firefly and Renilla luciferase activities were determined using Dual-luciferase reporter system according to the manufacturer's instructions (Promega, Madison, WI, USA). Luciferase activities were normalized to Renilla activities.

Western blot analysis. Tissues or cells were solubilized in cold radioimmunoprecipitation assay lysis buffer (Beyotime Institute of Biotechnology, Shanghai, China) and protein concentrations were measured by the standard BCA method $\left(\mathrm{BCA}^{\mathrm{TM}}\right.$ Protein Assay kit, USA). Equal amounts of protein were separated by $10 \%$ SDS-PAGE electronically and transferred onto a PVDF membrane (EMD Millipore, Billerica, MA, USA). Subsequently, the membranes were blocked with $5 \%$ non-fat dry milk in Tris-buffered saline with $0.05 \%$ Tween 20 (TBST) buffer and incubated overnight at $4^{\circ} \mathrm{C}$ with the following primary antibodies: mouse anti-human monoclonal RUNX3 antibody (sc-376591; 1:1,000 dilution; Santa Cruz Biotechnology, CA, USA) and mouse anti-human monoclonal GAPDH antibody (sc-47724; 1:1,000 dilution; Santa Cruz Biotechnology). Subsequent to washing three times with TBST, the membranes were incubated with goat anti-mouse
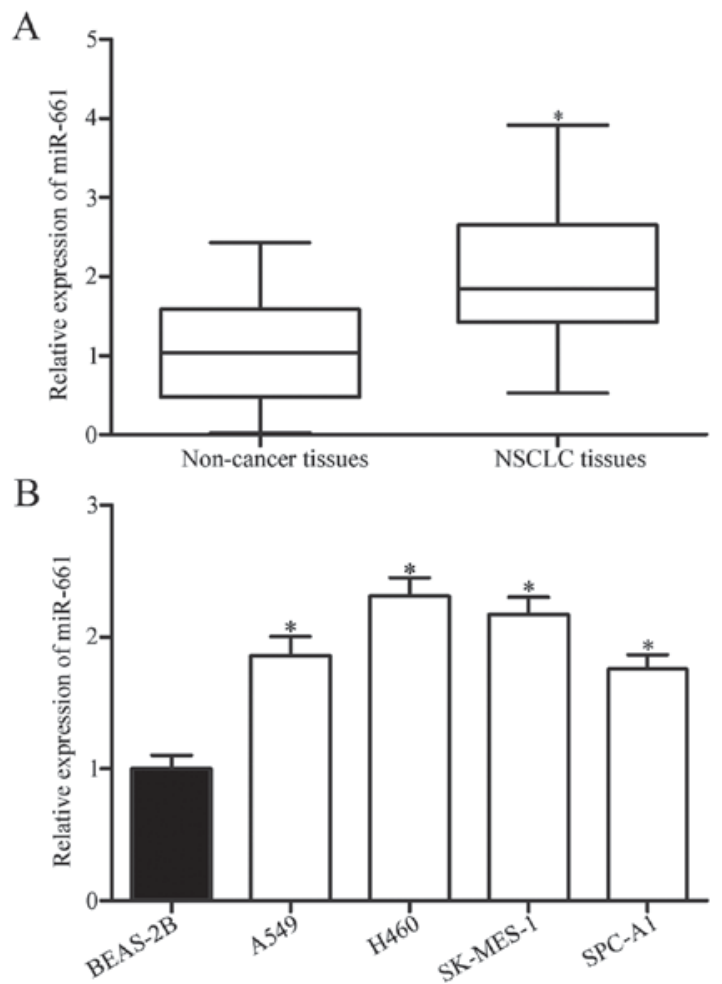

Figure 1. Expression level of miR-661 in NSCLC tissues and cell lines. (A) MiR-661 expression was determined in forty-seven pairs of NSCLC tissues and adjacent non-cancer tissues using RT-qPCR. "P<0.05 compared with non-cancer tissues. (B) Relative expression of miR-661 was detected in four NSCLC cell lines (A549, H460, SK-MES-1 and SPC-A1) and non-tumourigenic bronchial epithelium BEAS-2B cells. ${ }^{*} \mathrm{P}<0.05$ compared with BEAS-2B.

horseradish peroxidase (HRP)-conjugated secondary antibody (sc-2005; 1:5,000 dilution; Santa Cruz Biotechnology) at room temperature for $2 \mathrm{~h}$. The immunoblot was detected by an enhanced chemiluminescence solution (Pierce Biotechnology, Inc., Rockford, IL, USA). GAPDH was used as an internal control for RUNX3. Densitometric analysis was conducted using Image-Pro Plus software version 6.0 (Media Cybernetics, Inc., Rockville, MD, USA).

Statistical analysis. All data were presented as mean \pm standard errors. Statistical significance betwen groups were evaluated by Student's t-test or one-way analysis of variance (ANOVA).

SPSS 17.0 software (SPSS Inc., Chicago, IL, USA) was used for statistical analysis. $\mathrm{P}<0.05$ was considered statistically significant.

\section{Results}

MiR-661 is upregulated in NSCLC tissues and cell lines. To investigate whether or not miR-661 levels were altered in NSCLC, we measured miR-661 expression in 47 pairs of NSCLC tissues and adjacent non-cancer tissues through RT-qPCR. The results showed that miR-661 was significantly upregulated in NSCLC tissues compared with adjacent non-cancer tissues (Fig. $1 \mathrm{~A}, \mathrm{P}<0.05$ ). The association between miR-661 expression and clinicopathological factors of NSCLC patients was analysed. As shown in Table I, the miR-661 
A

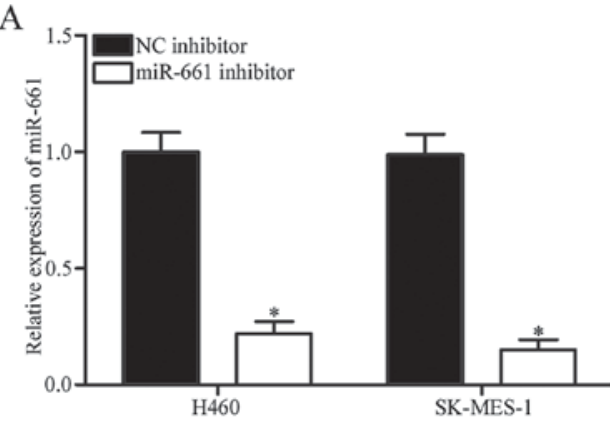

C

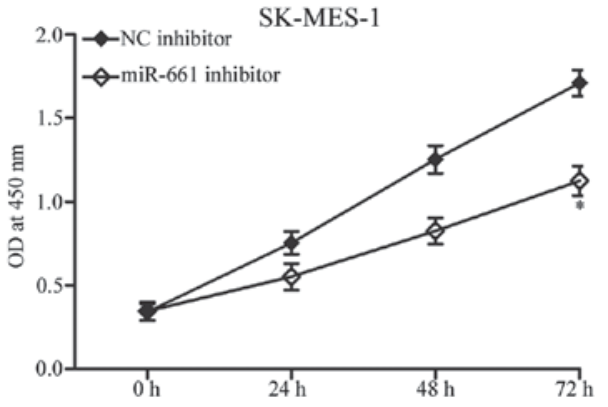

B

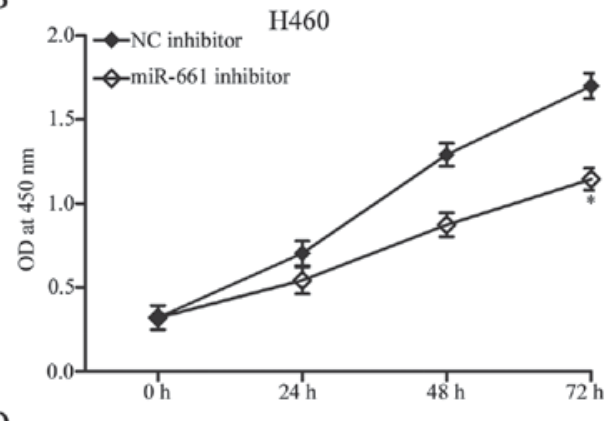

$\mathrm{D}$

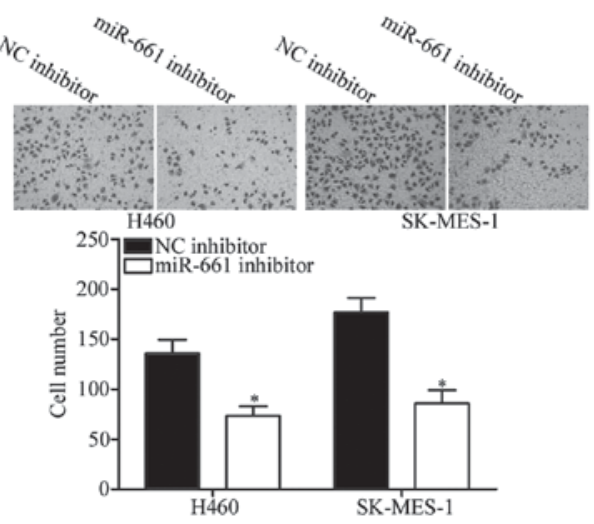

Figure 2. Downregulation of miR-661 suppressed H460 and SK-MES-1 cells proliferation and invasion. (A) RT-qPCR analysis of miR-661 expression levels in H460 and SK-MES-1 cells transfected with the miR-661 inhibitor or NC inhibitor. * $\mathrm{P}<0.05$ compared with NC inhibitor. (B and C) CCK8 assay was performed to evaluate the proliferation of $\mathrm{H} 460$ and SK-MES-1 cells transfected with miR-661 inhibitor or NC inhibitor. ${ }^{*} \mathrm{P}<0.05$ compared with NC inhibitor. (D) Cell invasion assay was used to determine the invasion ability of $\mathrm{H} 460$ and SK-MES-1 cells following transfection with miR-661 inhibitor or NC inhibitor. miR-661 underexpression significantly inhibited the invasiveness of $\mathrm{H} 460$ and SK-MES-1 cells. " $\mathrm{P}<0.05$ compared with NC inhibitor.

expression level was strongly correlated with differentiation $(\mathrm{P}=0.011)$, tumor stage $(\mathrm{P}=0.013)$ and lymph node metastasis $(\mathrm{P}=0.029)$, but not with gender, age, smoker and tumour size (all $\mathrm{P}>0.05$ ).

RT-qPCR was further carried out to quantify miR-661 expression levels in non-tumourigenic bronchial epithelium BEAS-2B cells and four NSCLC cell lines (A549, H460, SK-MES-1 and SPC-A1). Consistent with the results observed in clinical tissues, NSCLC cell line exhibited significantly higher miR-661 expression levels compared with BEAS-2B (Fig. 1B, $\mathrm{P}<0.05)$. These results suggested that miR-661 might play key roles in NSCLC formation and progression.

Downregulation of miR-661 suppresses NSCLC proliferation and invasion. To investigate the potential role of miR-661 in NSCLC, miR-661 inhibitor was transfected into H460 and SK-MES-1 cells, which expressed relatively higher miR-661 expression. RT-qPCR analysis confirmed that miR-661 was markedly downregulated in H460 and SK-MES-1 cells after transfection with miR-661 inhibitor (Fig. 2A, $\mathrm{P}<0.05$ ). The effect of miR-661 underexpression on NSCLC cell proliferation was evaluated using CCK-8 assay. As shown in Fig. 2B and C, miR-661 downregulation inhibited H460 and SK-MES-1 cell proliferation compared with NC inhibitor. We also assessed the ability of miR-661 to regulate NSCLC cell invasion using cell invasion assay. The results indicated that miR-661-underexpressed H460 and SK-MES-1 cells showed significantly less invasiveness than cells transfected with NC inhibitor (Fig. 2D, $\mathrm{P}<0.05$ ). These findings suggested that miR-661 may play an oncogenic role in NSCLC.
RUNX3 is a direct target of miR-661 in NSCLC. We sought to identify the direct target genes of miR-661 to explore the mechanism underlying its tumour-promoting activity in NSCLC. A considerable number of potential targets were predicted using bioinformatic analysis, and RUNX3 was selected for further confirmation (Fig. 3A) because it was downregulated in NSCLC tissues and contributed to NSCLC initiation and progression $(24,25)$. To determine whether RUNX3 is a direct target of miR-661, luciferase reporter assay was performed in H460 and SK-MES-1 cells co-transfected with psiCHECK2-RUNX3 3'UTR Wt or psiCHECK2-RUNX3 3'UTR Mut and miR-661 or NC inhibitor. As shown in Fig. 3B, co-transfection with miR-661 inhibitor increased the luciferase activities of wild-type RUNX3 3'-UTR plasmid compared with NC inhibitor $(\mathrm{P}<0.05)$. However, the luciferase activities of mutant RUNX3 3'-UTR constructs was not affected by miR-661 inhibitor. Further RT-qPCR and Western blot demonstrated that RUNX3 expression at both mRNA (Fig. 3C, P<0.05) and protein (Fig. 3D, $\mathrm{P}<0.05$ ) levels in $\mathrm{H} 460$ and SK-MES-1 cells was significantly increased after transfection with miR-661 inhibitor. These results revealed that RUNX3 is the direct target of miR-661 in NSCLC.

RUNX3 is downregulated and negatively correlated with miR-661 in NSCLC tissues. To further determine the relationship between miR-661 and RUNX3, RT-qPCR and Western blot were performed to measure RUNX3 expression at mRNA and protein levels in NSCLC tissues and adjacent non-cancer tissues. We found that RUNX3 expression level was decreased 
A

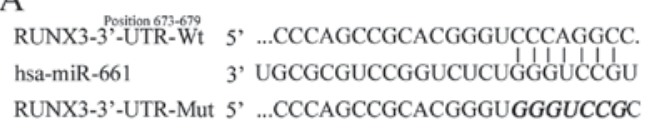
B

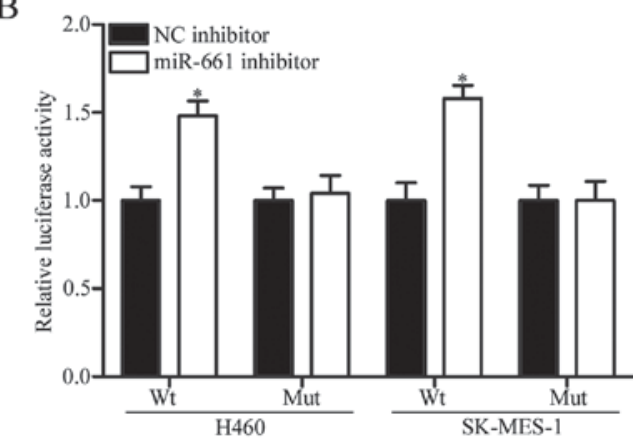

$\mathrm{C}$

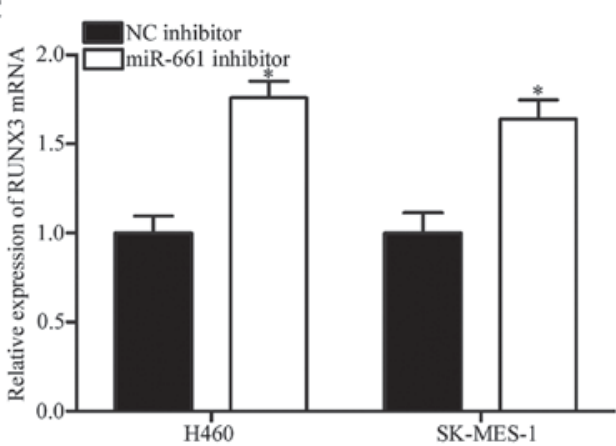

D

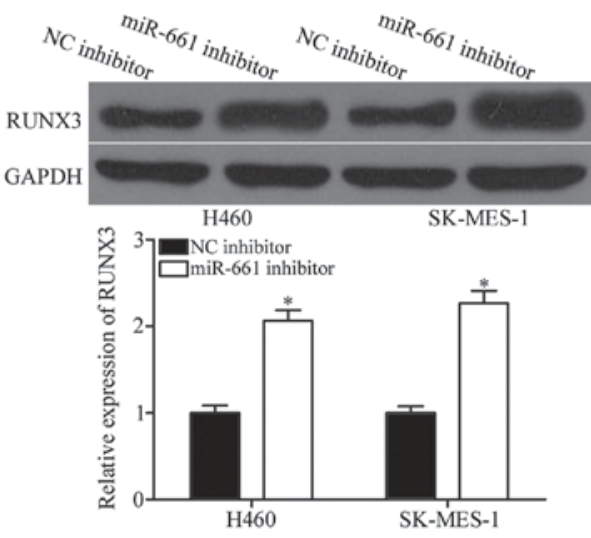

Figure 3. RUNX3 is a direct target of miR-661 in NSCLC. (A) Bioinformatic analysis predicted that the 3 -UTR of RUNX3 contains one miR-661 binding site. (B) Luciferase reporter assay of $\mathrm{H} 460$ and SK-MES-1 cells transfected with psiCHECK2-RUNX3 3'UTR Wt or psiCHECK2-RUNX3 3'UTR Mut and miR-661 inhibitor or NC inhibitor. Luciferase activities were measured $48 \mathrm{~h}$ after transfection. ${ }^{~} \mathrm{P}<0.05$ compared with NC inhibitor. (C and D) RUNX3 mRNA and protein expression in H460 and SK-MES-1 cells transfected with miR-661 inhibitor or NC inhibitor was detected through RT-qPCR and Western blot, respectively. ${ }^{*}<<0.05$ compared with $\mathrm{NC}$ inhibitor.

at both mRNA (Fig. 4A, P<0.05) and protein (Fig. 4B, P<0.05) levels in NSCLC tissues compared with that in adjacent non-cancer tissues. Additionally, we assessed the association between RUNX3 mRNA and miR-661 expression levels in NSCLC tissues. The results of Spearman's correlation analysis indicated a statistically inverse association between RUNX3 mRNA and miR-661 expression levels in NSCLC tissues (Fig. 4C; $r=-0.6960, \mathrm{P}<0.0001$ ).

RUNX3 knockdown rescued the effects induced by miR-661 inhibitor on NSCLC cells. Rescue experiments were carried

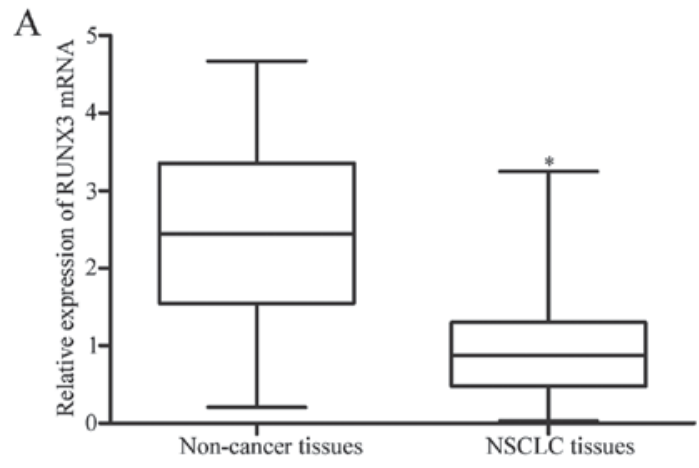

B

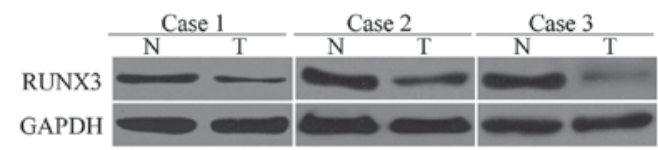

$\mathrm{C}$

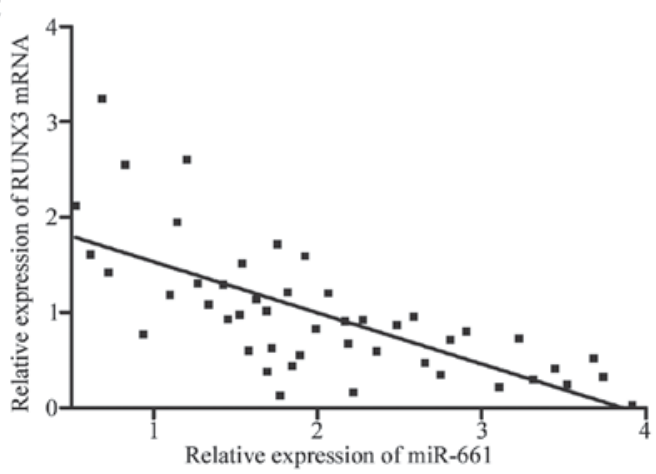

Figure 4. RUNX3 was downregulated and inversely correlated with miR-661 expression in NSCLC tissues. (A and B) RT-qPCR of RUNX3 mRNA and Western blot of RUNX3 protein in NSCLC tissues and adjacent non-cancer tissues. * $\mathrm{P}<0.05$ compared with non-cancer tissues. (C) Spearman's correlation analysis was adopted to investigate the association between RUNX3 mRNA and miR-661 expression in NSCLC tissues. $\mathrm{r}=-0.6960, \mathrm{P}<0.0001$.

out to explore whether or not RUNX3 is responsible for the functional effect of miR-661 in NSCLC cells. Si-RUNX3 or si-NC was introduced into H460 and SK-MES-1 cells, and Western blot confirmed that RUNX3 was downregulated in si-RUNX3-transfected H460 and SK-MES-1 cells (Fig. 5A, $\mathrm{P}<0.05$ ). Subsequently, CCK-8 and cell invasion assays were performed, which identified that RUNX3 knockdown significantly rescued the effects of miR-661 inhibitor on $\mathrm{H} 460$ and SK-MES-1 cell proliferation (Fig. 5B and C, P<0.05) and invasion (Fig. 5D, $\mathrm{P}<0.05$ ). These findings suggested that miR-661 exerts its biological roles in NSCLC through negative regulation of RUNX3.

\section{Discussion}

Increasing evidence indicated that abnormal miRNA expression contributes to the carcinogenesis and progression of multiple human cancers (26-28). Numerous studies have shed light on tumour-targeting therapies using miRNAs as novel diagnostic and therapeutic tools (29-31). Therefore, elucidating the expression level, clinical significance, biological roles and underlying mechanisms of specific miRNA in NSCLC will provide novel therapeutic targets for the diagnosis and therapy of patients with this disease. In the present study, we found that the miR-661 expression was upregulated in NSCLC tissues 
A

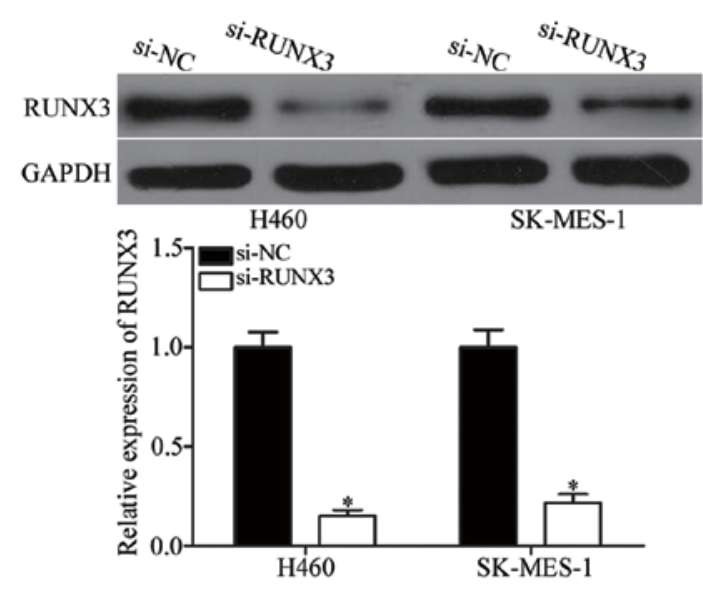

C

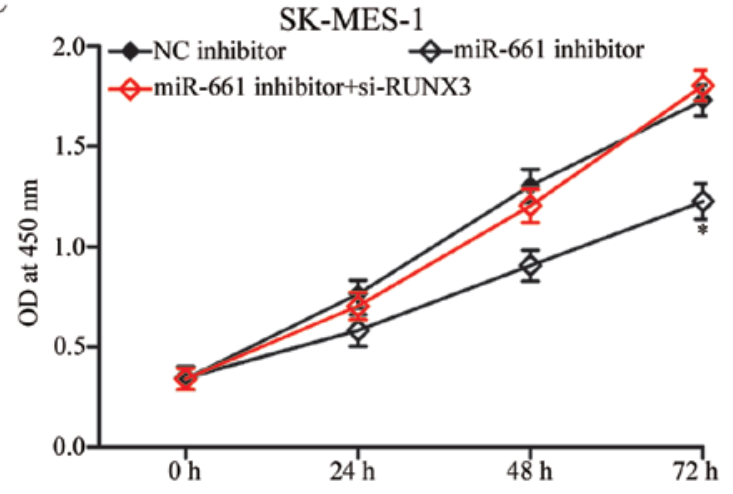

B

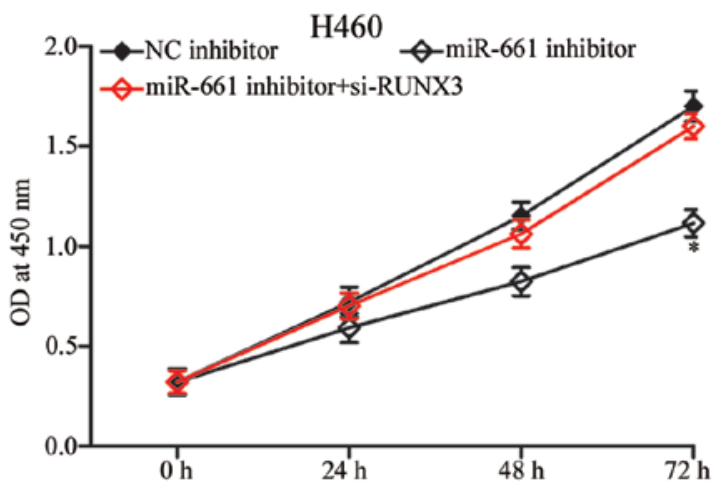

$\mathrm{D}$

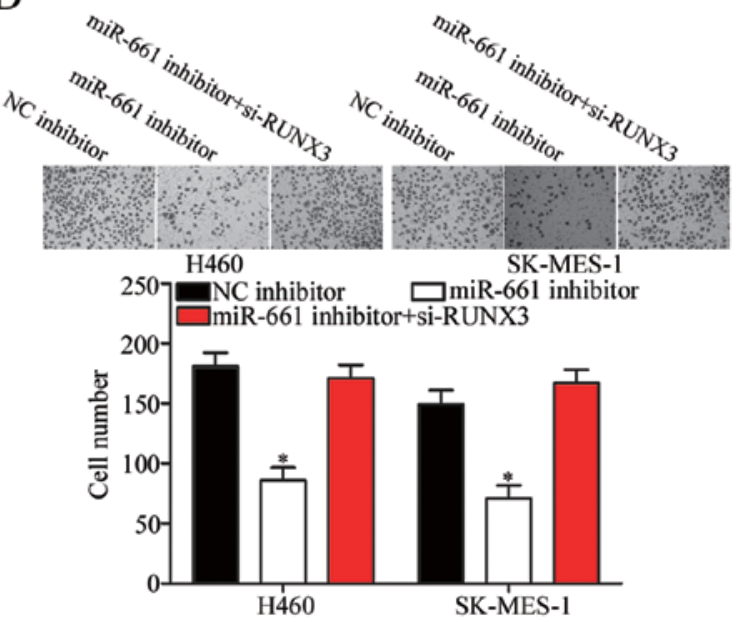

Figure 5. RUNX3 knockdown reverses the effects of miR-661 inhibitor on H460 and SK-MES-1 cells proliferation and invasion. (A) Western blot analysis indicated that RUNX3 protein in $\mathrm{H} 460$ and SK-MES-1 cells was downregulated following transfection with si-RUNX3. ${ }^{*}<0.05$ compared with si-NC. (B-D) CCK-8 and cell invasion assays detected the proliferation and invasion of H460 and SK-MES-1 cells transfected with NC inhibitor or miR-661 inhibitor or miR-661 inhibitor co-transfected with si-RUNX3. ${ }^{*} \mathrm{P}<0.05$ compared with NC inhibitor and miR-661 inhibitor+si-RUNX3.

and cell lines. The miR-661 expression level was significantly correlated with differentiation, tumor stage and lymph node metastasis of NSCLC patients. Functional assays indicated that miR-661 underexpression attenuated NSCLC cell proliferation and invasion in vitro. With regard to the mechanism, our results demonstrated that RUNX3 functions as a direct downstream target of miR-661 in NSCLC. These results demonstrated that miR-661 may play significant roles in NSCLC.

Previously, miR-661 was revealed to be aberrantly expressed in a number of human malignancies and play a crucial role in tumourigenesis and cancer progression. For example, Li et al (20) reported that miR-661 was lowly expressed in glioma tissues. Restoration of miR-661 expression inhibited glioma cell proliferation, migration and invasion; induced cell cycle arrest and apoptosis in vitro; and decreased cell growth in vivo. Zhu et al (21) found that miR-661 was upregulated in both ovarian cancer tissues and cell lines. Upregulation of miR-661 promoted cell proliferation, colony formation and anchorage-independent growth in vitro. Reddy et al (22) demonstrated that ectopic miR-661 expression repressed cell migration, invasion and anchorage-independent growth and tumourigenicity of breast cancer. These findings indicated that miR-661 may be investigated as an effective therapeutic target for these types of cancer.

The identification of the direct target gene of miR-661 is important in understanding its roles in NSCLC and is also essential in the investigation of novel therapeutic target for NSCLC patients. Several targets of miR-661 have been reported, including hTERT (20) in glioma, INPP5J (21) in ovarian cancer and MTA1 (22) in breast cancer. In the present study, RUNX3 was identified as a direct target gene of miR-661 in NSCLC. Initially, the bioinformatic analysis revealed that RUNX3 may be a potential target for miR-661. Subsequently, this predication was further confirmed by the luciferase report assay. The results demonstrated that the 3'-UTR of RUNX3 could be directly targeted by miR-661 in NSCLC cells. Notably, the regulatory effect of miR-661 on endogenous RUNX3 expression in NSCLC cells was determined using RT-qPCR and western blot, and demonstrated that downregulation of miR-661 significantly increased RUNX3 expression at the mRNA and protein levels in NSCLC cells. Besides, RUNX3 was downregulated and negative correlated with miR-661 expression level in NSCLC tissues. Finally, RUNX3 knockdown rescued the effects observed as a result of miR-661 underexpression in NSCLC cells.

Runt-related gene family includes three members, namely, RUNX1, RUNX2, and RUNX3, and all play key roles in the normal developmental process and tumourigenesis (32). RUNX3, which is located on chromosome 1p36, has been reported to be downregulated in many human cancer types, such as bladder cancer (33), oesophageal squamous cell 
carcinoma (34), breast cancer (35), gastric cancer (36) and glioma (32). In addition, RUNX3 is regarded as a tumour suppressor gene that inhibits cell proliferation, migration and invasion and induces cell cycle arrest and apoptosis (37-39). Previous studies also found that RUNX3 was reduced in NSCLC tissues, which was associated with promoter hypermethylation (24). Additionally, RUNX3 methylation was obviously correlated with NSCLC clinical stage, lymph node metastasis and differentiation (40). Functional experiments revealed that RUNX3 is involved in crucial regulation of cell proliferation (41), epithelial-mesenchymal transition (42) and tumourigenesis (25) in NSCLC. These findings indicated that RUNX3 may contribute to NSCLC progression. The present study identified RUNX3 as a direct gene target of miR-661, suggesting that the miR-661/RUNX3 axis may be a promising therapeutic target for the treatment of NSCLC patients.

In conclusion, we found that miR-661 expression level was increased in NSCLC tissues and cell lines. In addition, miR-661 expression level was significantly correlated with differentiation, tumor stage and lymph node metastasis of NSCLC patients. Furthermore, miR-661 acted as an oncogene in NSCLC, at least in part through RUNX3 targeting. These observations suggest that miR-661 may serve as a novel therapeutic target for the treatment of NSCLC.

\section{References}

1. Jemal A, Siegel R, Xu J and Ward E: Cancer statistics, 2010. CA Cancer J Clin 60: 277-300, 2010.

2. Torre LA, Bray F, Siegel RL, Ferlay J, Lortet-Tieulent J and Jemal A: Global cancer statistics, 2012. CA Cancer J Clin 65: 87-108, 2015.

3. Cushing L, Jiang Z, Kuang P and Lü J: The roles of microRNAs and protein components of the microRNA pathway in lung development and diseases. Am J Respir Cell Mol Biol 52: 397-408, 2015.

4. Ebrahimi A and Sadroddiny E: MicroRNAs in lung diseases: Recent findings and their pathophysiological implications. Pulm Pharmacol Ther 34: 55-63, 2015

5. DeSantis CE, Lin CC, Mariotto AB, Siegel RL, Stein KD, Kramer JL, Alteri R, Robbins AS and Jemal A: Cancer treatment and survivorship statistics, 2014. CA Cancer J Clin 64: 252-271, 2014.

6. Monirul Islam KM, Shostrom V, Kessinger A and Ganti AK: Outcomes following surgical treatment compared to radiation for stage I NSCLC: A SEER database analysis. Lung Cancer 82: 90-94, 2013.

7. Xue C, Hu Z, Jiang W, Zhao Y, Xu F, Huang Y, Zhao H, Wu J, Zhang Y, Zhao L, et al: National survey of the medical treatment status for non-small cell lung cancer (NSCLC) in China. Lung Cancer 77: 371-375, 2012.

8. Laskin JJ and Sandler AB: State of the art in therapy for non-small cell lung cancer. Cancer Invest 23: 427-442, 2005.

9. Bartel DP: MicroRNAs: Genomics, biogenesis, mechanism, and function. Cell 116: 281-297, 2004.

10. Ambros V: The functions of animal microRNAs. Nature 431: 350-355, 2004

11. Huang Q, Xiao B, Ma X, Qu M, Li Y, Nagarkatti P, Nagarkatti M and Zhou J: MicroRNAs associated with the pathogenesis of multiple sclerosis. J Neuroimmunol 295-296: 148-161, 2016.

12. Calin GA, Sevignani C, Dumitru CD, Hyslop T, Noch E, Yendamuri S, Shimizu M, Rattan S, Bullrich F, Negrini M and Croce CM: Human microRNA genes are frequently located at fragile sites and genomic regions involved in cancers. Proc Natl Acad Sci USA 101: 2999-3004, 2004.

13. Meltzer PS: Cancer genomics: Small RNAs with big impacts. Nature 435: 745-746, 2005.

14. Bao L, Wang L, Wei G, Wang Y, Wuyun G and Bo A: Role of microRNA-4458 in patients with non-small-cell lung cancer. Oncol Lett 12: 3958-3966, 2016.
15. Hu W, Jin P, Ding C and Liu W: miR-19a/b modulates lung cancer cells metastasis through suppression of MXD1 expression. Oncol Lett 12: 1901-1905, 2016.

16. Sahay D, Leblanc R, Grunewald TG, Ambatipudi S, Ribeiro J, Clézardin P and Peyruchaud O: The LPA1/ZEB1/miR-21-activation pathway regulates metastasis in basal breast cancer. Oncotarget 6: 20604-20620, 2015.

17. Maugeri-Saccà M, Coppola V, Bonci D and De Maria R: MicroRNAs and prostate cancer: From preclinical research to translational oncology. Cancer J 18: 253-261, 2012.

18. Jones KB, Salah Z, Del Mare S, Galasso M, Gaudio E, Nuovo GJ, Lovat F, LeBlanc K, Palatini J, Randall RL, et al: miRNA signatures associate with pathogenesis and progression of osteosarcoma. Cancer Res 72: 1865-1877, 2012.

19. Bartels CL and Tsongalis GJ: MicroRNAs: Novel biomarkers for human cancer. Clin Chem 55: 623-631, 2009.

20. Li Z, Liu YH, Diao HY, Ma J and Yao YL: MiR-661 inhibits glioma cell proliferation, migration and invasion by targeting hTERT. Biochem Biophys Res Commun 468: 870-876, 2015.

21. Zhu T, Yuan J, Wang Y, Gong C, Xie Y and Li H: MiR-661 contributed to cell proliferation of human ovarian cancer cells by repressing INPP5J expression. Biomed Pharmacother 75: 123-128, 2015.

22. Reddy SD, Pakala SB, Ohshiro K, Rayala SK and Kumar R: MicroRNA-661, a c/EBPalpha target, inhibits metastatic tumor antigen 1 and regulates its functions. Cancer Res 69: 5639-5642, 2009.

23. Livak KJ and Schmittgen TD: Analysis of relative gene expression data using real-time quantitative PCR and the 2(-Delta Delta C(T)) Method. Methods 25: 402-408, 2001.

24. Lee YS, Lee JW, Jang JW, Chi XZ, Kim JH, Li YH, Kim MK, Kim DM, Choi BS, Kim EG, et al: Runx3 inactivation is a crucial early event in the development of lung adenocarcinoma. Cancer Cell 24: 603-616, 2013.

25. Lee KS, Lee YS, Lee JM, Ito K, Cinghu S, Kim JH, Jang JW, $\mathrm{Li} \mathrm{YH}$, Goh YM, Chi XZ, et al: Runx3 is required for the differentiation of lung epithelial cells and suppression of lung cancer. Oncogene 29: 3349-3361, 2010.

26. Xiao CZ, Wei W, Guo ZX, Zhang MY, Zhang YF, Wang JH, Shi M, Wang HY and Guo RP: MicroRNA-34c-3p promotes cell proliferation and invasion in hepatocellular carcinoma by regulation of NCKAP1 expression. J Cancer Res Clin Oncol 143: 263-273, 2017.

27. Jiang $\mathrm{H}, \mathrm{Ju} \mathrm{H}$, Zhang L, Lu H and Jie K: microRNA-577 suppresses tumor growth and enhances chemosensitivity in colorectal cancer. J Biochem Mol Toxicol: Feb 2, 2017 (Epub ahead of print).

28. Wang H, Li Q, Niu X, Wang G, Zheng S, Fu G and Wang Z: miR-143 inhibits bladder cancer cell proliferation and enhances their sensitivity to gemcitabine by repressing IGF-1R signaling. Oncol Lett 13: 435-440, 2017.

29. Gandellini P, Giovannetti E and Nicassio F: MicroRNAs in cancer management: Big challenges for small molecules. Biomed Res Int 2015: 982156, 2015.

30. Mlcochova J, Faltejskova-Vychytilova P, Ferracin M, Zagatti B, Radova L, Svoboda M, Nemecek R, John S, Kiss I, Vyzula R, et al: MicroRNA expression profiling identifies miR-31-5p/3p as associated with time to progression in wild-type RAS metastatic colorectal cancer treated with cetuximab. Oncotarget 6: 38695-38704, 2015.

31. Parpart S, Roessler S, Dong F, Rao V, Takai A, Ji J, Qin LX, Ye QH, Jia HL, Tang ZY and Wang XW: Modulation of miR-29 expression by $\alpha$-fetoprotein is linked to the hepatocellular carcinoma epigenome. Hepatology 60: 872-883, 2014.

32. Mei PJ, Bai J, Liu H, Li C, Wu YP, Yu ZQ and Zheng JN: RUNX3 expression is lost in glioma and its restoration causes drastic suppression of tumor invasion and migration. J Cancer Res Clin Oncol 137: 1823-1830, 2011.

33. Dodurga Y, Avci CB, Satiroglu-Tufan NL, Tataroglu C, Kesen Z, Doğan ZO, Yilmaz $S$ and Gündüz C: Detection of deleted in malignant brain tumors 1 and runt-related transcription factor 3 gene expressions in bladder carcinoma. Mol Biol Rep 39: 4691-4695, 2012.

34. Sugiura H, Ishiguro H, Kuwabara Y, Kimura M, Mitsui A, Mori Y, Ogawa R, Katada T, Harata K and Fujii Y: Decreased expression of RUNX3 is correlated with tumor progression and poor prognosis in patients with esophageal squamous cell carcinoma. Oncol Rep 19: 713-719, 2008.

35. Jiang Y, Tong D, Lou G, Zhang Y and Geng J: Expression of RUNX3 gene, methylation status and clinicopathological significance in breast cancer and breast cancer cell lines. Pathobiology 75: 244-251, 2008. 
36. Hsu PI, Hsieh HL, Lee J, Lin LF, Chen HC, Lu PJ and Hsiao M Loss of RUNX3 expression correlates with differentiation, nodal metastasis, and poor prognosis of gastric cancer. Ann Surg Oncol 16: 1686-1694, 2009.

37. Jili S, Eryong L, Lijuan L and Chao Z: RUNX3 inhibits laryngeal squamous cell carcinoma malignancy under the regulation of miR-148a-3p/DNMT1 axis. Cell Biochem Funct 34: 597-605, 2016.

38. Chen F, Liu X, Cheng Q, Zhu S, Bai J and Zheng J: RUNX3 regulates renal cell carcinoma metastasis via targeting miR-6780a-5p/E-cadherin/EMT signaling axis. Oncotarget: Nov 8, 2016 (Epub ahead of print).

39. Kim BR, Kang MH, Kim JL, Na YJ, Park SH, Lee SI, Kang S, Joung SY, Lee SY, Lee DH, et al: RUNX3 inhibits the metastasis and angiogenesis of colorectal cancer. Oncol Rep 36: 2601-2608, 2016.
40. Yu GP, Ji Y, Chen GQ, Huang B, Shen K, Wu S and Shen ZY: Application of RUNX3 gene promoter methylation in the diagnosis of non-small cell lung cancer. Oncol Lett 3: 159-162, 2012.

41. Torshabi M, Faramarzi MA, Tabatabaei Yazdi M, Ostad SN and Gharemani MH: Runx3 expression inhibits proliferation and distinctly alters mRNA expression of Bax in AGS and A549 cancer cells. Iran J Pharm Res 10: 355-361, 2011.

42. Lee JM, Shin JO, Cho KW, Hosoya A, Cho SW, Lee YS, Ryoo HM, Bae SC and Jung HS: Runx3 is a crucial regulator of alveolar differentiation and lung tumorigenesis in mice. Differentiation 81: 261-268, 2011. 